\title{
A CLASS OF MAPS IN AN ALGEBRA WITH INDEFINITE METRIC
}

\author{
ANGELO B. MINGARELLI
}

(Communicated by Palle E. T. Jorgensen)

Dedicated to the memory of David Conibear

\begin{abstract}
We study a class of hermitian maps on an algebra endowed with an indefinite inner product. We show that, in particular, the existence of a nonreal eigenvalue is incompatible with the existence of a real eigenvalue having a right-invertible eigenvector. It also follows that for this class of maps the existence of an appropriate extremal for an indefinite Rayleigh quotient implies the nonexistence of nonreal eigenvalues. These results are intended to complement the Perron-Fröbenius and Krein-Rutman theorems, and we conclude the paper by describing applications to ordinary and partial differential equations and to tridiagonal matrices.
\end{abstract}

\section{INTRODUCTION}

Let $(K,[]$,$) be an indefinite inner product algebra over \mathbb{C}$ with identity, $e$; that is, $K$ is an algebra and [ , ]: $K \times K \rightarrow \mathbb{C}$ is a sesquilinear hermitian (symmetric) form which is indefinite on $K$ (i.e., there exists $x, y \in K$ with $[x, x]>0$ and $[y, y]<0)$. Thus $K$ is, in particular, an indefinite inner product space $[4$, p. 3].

In the sequel $A: \mathscr{D}(A) \subseteq K \rightarrow K, \mathscr{D}(A) \neq \varnothing$, is a nontrivial hermitian map, i.e.,

$$
[A f, g]=[f, A g], \quad f, g \in \mathscr{D}(A),
$$

and $\psi \neq 0$ denotes a right-invertible element in $K$. Note that $A$ is not necessarily linear (since $\mathscr{D}(A)$ may be a discrete set in an infinite-dimensional space). Furthermore, $I$ is the identity map and "multiplication by $\varphi$ " is defined by $(\phi I)(u)=\varphi u, u \in K$. We always assume that the inner product [ , ] is indefinite on $\mathscr{D}(A)$. The map $A$ is homogeneous if, for every $m \in \mathbb{C}$, $m \neq 0, A(m u)=m A u, u \in \mathscr{D}(A)$.

We study maps $A$ on $K$ with the property that, whenever the display is real,

$$
\left[\left(A-(A \psi) \psi^{-1} I\right) u, u\right]>0
$$

Received by the editors November 9,1992 ; presented during a special session talk at the conference Operator Theory and Boundary Eigenvalue Problems, Technical University of Vienna, Vienna, Austria, August 1993.

1991 Mathematics Subject Classification. Primary 46C20, 47B50; Secondary 34C10, 34B24, 35P05, 39A70, 15A18.

Key words and phrases. Krein space, hermitian maps, Perron-Fröbenius.

Partially funded by a grant from N.S.E.R.C. Canada. 
for every $u \in \mathscr{D}(A)$ and $\psi \in \mathscr{I}$ and $\mathscr{I}$ is some collection of right-invertible elements from $K$ (thus $\psi \psi^{-1}=e, \psi \in \mathscr{I}$ ).

The class $\mathscr{S}(\psi)$ denotes the collection of all maps $A$ which for a given $\mathscr{F}$ satisfy (1.1) for $\psi \in \mathscr{I}$ and for which

$$
\left[\left(A-(A \psi) \psi^{-1} I\right) u, u\right]=0
$$

if and only if $u \in\langle\psi\rangle$ (the linear span of $\psi$ ).

The main results of this paper relate the existence of nonreal eigenvalues of maps $A \in \mathscr{S}(\psi)$ to the nonexistence of a corresponding real eigenvalue having a right-invertible eigenvector. In this sense, the phenomenon is in contrast and complementary to the classical Perron-Fröbenius theorem for matrices and the more general Krein-Rutman theorem [5, p. 74], each of which guarantees, in its own setting and under appropriate conditions, the existence of a real eigenvalue having an eigenvector in a suitable cone and such even in the presence of nonreal eigenvalues. We interpret our results in the language of cones when $K$ is a Krein space $\left[4\right.$, p. 100] of $L^{2}$ functions on a finite interval. We apply the various abstract results obtained to the cases where $A$ is a Sturm-Liouville operator with an indefinite weight-function acting on a weighted $L^{2}$-space (actually a Krein space) and thereby recover results from [1, p. 4]. One may think of (1.1) as an abstract "Picone Identity" of sorts.

\section{THE MAIN RESULTS}

Theorem 2.1. Let $\mathscr{I} \neq \phi$ be given, and let $A$ be a homogeneous nontrivial hermitian map on the indefinite inner product algebra $(K[]$,$) , over \mathbb{C}$, with identity. If $A \in \mathscr{S}(\psi)$ for every $\psi \in \mathscr{I}$ and $A$ has a nonreal eigenvalue, then $A$ has no real eigenvalue with a corresponding right-invertible eigenvector $\varphi$ in $\mathscr{I}$.

Proof. Let $A u=\mu u$ where $\operatorname{Im}(\mu) \neq 0$ and $u \neq 0$ is a corresponding eigenvector. Then $[A u, u]=0$ and $[u, u]=0$.

Assume, on the contrary, that $\varphi \in \mathscr{J}$ is a right-invertible eigenvector of $A$ corresponding to a real eigenvalue $\lambda$. Since $A \in \mathscr{S}(\varphi)$, it follows that, if $u \in \mathscr{D}(A)$,

$$
\begin{aligned}
{\left[\left(A-(A \varphi) \varphi^{-1} I\right) u, u\right] } & =[A u, u]-\left[(A \varphi) \varphi^{-1} I u, u\right] \\
& =-[\lambda e I u, u]=-\lambda[u, u]=0
\end{aligned}
$$

therefore, $u \in\langle\varphi\rangle$, i.e., $u=m \varphi, m \neq 0$, in $\mathbb{C}$. Next, $m(\lambda \varphi)=m A \varphi=$ $A(m \varphi)=\mu(m \varphi)$, so $m(\lambda-\mu) \varphi=0$, i.e., $\mu$ must be real, a contradiction.

Remarks. 1. It follows from the proof that one can show the slightly more general result: If $A$ has an eigenvalue (possibly real) whose corresponding eigenvector $u$ satisfies $[A u, u]=0=[u, u]$, then $A$ cannot have a real eigenvalue with a right-invertible eigenvector except possibly for $u$ itself.

2. A need not be linear. For example, let $(K,[]$,$) be an indefinite inner$ product space over $\mathbb{C}$, and choose $x \neq 0$ such that $[x, x]=0$ (see [4, Lemma 2.1, p. 4]). Define $A$ by $A 0=x$ and $A(c x)=0$ for $c \neq 0$ in $\mathbb{C}$. Then $A$ is hermitian on $\mathscr{D}(A)=\langle x\rangle, A(c x)=c A x$ for $c \neq 0$, but $A$ is not linear.

3. A "cone" analog of Theorem 2.1 in Krein space follows: Here $K$ is the Krein space of functions in the complex Hilbert space $L^{2}(-1,1)$ with the 
indefinite inner product

$$
[f, g]=\int_{-1}^{1} f(x) \bar{g}(x)(\operatorname{sgn} x) d x
$$

where $\operatorname{sgn} x= \pm 1$, according as to whether $x>0, x<0$.

Let $\mathscr{I}$ be the collection of all positive continuous functions on $(-1,1)$. Then $\mathscr{I}$ is a cone in $K$. Indeed, every such element is invertible in the subalgebra generated by $\mathscr{I}$ (under the usual operations).

It is not difficult to see that the inner product [, ] is indefinite on $\mathscr{I}$ as well. So under the appropriate conditions on $A$ we can deduce the nonexistence of positive solutions of $A \psi=\lambda \psi$ for $\lambda \in \mathbb{R}$ (cf. [1, p. 4; 2, p. 70]).

4. The assumption that $A \in \mathscr{S}$ in Theorem 2.1 cannot be waived in general since there exists a (linear) symmetric operator on the Krein space of Remark 3 , whose spectrum in all of $\mathbb{C}$ and consists only of eigenvalues. Among these there is an interval of (real) eigenvalues with the property that associated eigenfunctions have no zeros in $(-1,1)$ [3, p. 381]. Incidentally we note, in passing, that a symmetric operator in an indefinite inner product space may have nonreal eigenvalues [4, Example 3.2, p. 35].

5. The hypothesis that $A$ satisfies (1.1) is not used in the proof, and therefore Theorem 2.1 is valid for $A$ 's merely satisfying (1.2).

\section{Applications}

In this section we apply the foregoing results to ordinary and partial differential equations and to tridiagonal matrices.

We shall assume, unless otherwise specified, that the sets $E^{+}=\{x \in[a, b]$ : $r(x)>0\}$ and $\left.E^{-}=\{x \in a, b]: r(x)<0\right\}$ have positive Lebesgue measure (denoted by $\lambda$ ) and that $\lambda\left(E^{0}\right)=0$ where $E^{0}=\{x \in[a, b]: r(x)=0\}$. Here $r:[a, b] \rightarrow \mathbb{R}, r \in L(a, b)$, where $-\infty<a<b<\infty$. The indefinite inner product [, ] is defined on the weighted complex Hilbert space $L_{|r|}^{2}(a, b)=K$ by

$$
[f, g]=\int_{a}^{b} f(x) \bar{g}(x) r(x) d x
$$

for $f, g \in K$. As is usual, $K$ denotes the space of all Lebesgue measurable functions which are square integrable with respect to the measure $|r(x)| d x$. The inner product $($,$) on K$ is related to [, ] via

$$
[f, g]=(J f, g)
$$

where $(J f)(x)=(\operatorname{sgn} r(x)) f(x)$ is a multiplication operator, $J^{2}=I$, and $J$ is completely invertible [4, p. 100].

The space $K$ thus endowed with the inner product [, ] is a Krein space $[4$, p. 100] that is also an indefinite inner product (commutative) algebra with identity (provided the functions are, e.g., bounded).

We now fix $q \in L(a, b), q:[a, b] \rightarrow \mathbb{R}$ and define an operator $A$ by

$$
(A f)(x)=\left\{-f^{\prime \prime}(x)+q(x) f(x)\right\} / r(x)
$$

with domain

$$
\begin{aligned}
\mathscr{D}(A)=\{f \in K: & f, f^{\prime} \in A C[a, b], \quad A f \in K, \text { and } \\
& \left.f(a)=f(b)=0, f^{\prime}(a) \neq 0, f^{\prime}(b) \neq 0\right\} .
\end{aligned}
$$


A straightforward calculation shows that

$$
[A f, g]=[f, A g], \quad f, g \in \mathscr{D}(A),
$$

so that $A$ is hermitian on $K$. It is known that, in particular situations $A$ may have nonreal eigenvalues $[1,2,3]$.

Consider the space $\mathscr{J}$ of functions in $K$ defined by

$$
\mathscr{I}=\{f:[a, b] \rightarrow \mathbb{R}, f \in \mathscr{D}(A), f(x)>0 \text { in }(a, b)\} .
$$

Then $\mathscr{I}$ is a collection of (right-)invertible elements of the algebra $K$.

Theorem 3.1. For $\psi \in \mathscr{I}, A \in \mathscr{S}(\psi)$.

Proof. For $u \in \mathscr{D}(A), u \neq 0$, suppressing the independent variables for clarity, we have

$$
\begin{aligned}
{\left[\left(A-(A \psi) \psi^{-1} I\right) u, u\right] } & =[A u, u]-\left[(A \psi) \psi^{-1} u, u\right] \\
& =\int_{a}^{b}\left(\left|u^{\prime}\right|^{2}+q|u|^{2}\right) d x+\int_{a}^{b} \frac{\psi^{\prime \prime}|u|^{2}}{\psi} d x-\int_{a}^{b} q|u|^{2} d x \\
& =\int_{a}^{b}\left\{\left|u^{\prime}\right|^{2}+\frac{|u|^{2} \psi^{\prime 2}}{\psi^{2}}-\frac{2 \psi^{\prime}}{\psi} \operatorname{Re}\left(u \bar{u}^{\prime}\right)\right\} d x \\
& =\int_{a}^{b}\left|u^{\prime}-\frac{u \psi^{\prime}}{\psi}\right|^{2} d x .
\end{aligned}
$$

It now follows from the last display that (1.2) holds for $u \in\langle\psi\rangle$ and conversely. Remark. A similar argument may be used, with the necessary changes, in order to show that the weighted Schrödinger operator

$$
(A f)(x)=\{-\Delta f(x)+q(x) f(x)\} / r(x),
$$

on a smooth bounded domain $\Omega \subset \mathbb{R}^{n}, n>1$, and $q, r \in L^{\infty}(\Omega)$ with $r(x)$ vanishing only on sets of zero measure and defined on a suitable (operator) domain $\mathscr{D}(A)$ which ensures that

$$
\int_{\partial \Omega} \frac{u \bar{u}}{\psi} \frac{\partial \psi}{\partial n} d \sigma=0, \quad u \in \mathscr{D}(A), \psi \in \mathscr{I}
$$

(corresponding to the case $n=1$ ), is also in $\mathscr{S}(\psi)$.

Corollary 3.1. If

$$
\min _{\substack{u \in \mathscr{D}(A) \\[u, u] \neq 0}} \frac{[A u, u]}{[u, u]}=\lambda_{0}
$$

exists and is attained only for $u(x)=C \psi(x)$ where $A \psi=\lambda_{0} \psi$ and $\psi \in \mathscr{I}$, then $A$ has no nonreal eigenvalues.

Proof. The assumption implies that

$$
\left[\left(A-\lambda_{0} I\right) u, u\right] \geq 0
$$

with equality iff $u \in\langle\psi\rangle$, which in turn is equivalent to

$$
\left[\left(A-(A \psi) \psi^{-1} I\right) u, u\right] \geq 0
$$

with equality iff $u \in\langle\psi\rangle$ which, of course, implies (1.2). The result is now a consequence of Theorems 3.1 and 2.1. 
Remark. This corollary is surprising in that it ensures the variational characterization of $\lambda_{0}$, as defined there, only if $A$ has no nonreal eigenvalues.

By analogy, it is well known that if $r(x)>0$ a.e. on $[a, b]$, then $K$ is, in fact, a Hilbert space and not a Krein space and $\lambda_{0}$ is then the smallest eigenvalue of $A$ (to which there corresponds a positive eigenfunction). It is also clear in this case that $A$ has real spectrum only.

We now proceed with a final application to matrix theory; this is by far the most technical application.

In the sequel $\mathbb{C}^{m}, m \geq 2$, is endowed with the usual inner product $($, and the indefinite inner product [ , ] defined by $f=\left(f_{n}\right), g=\left(g_{n}\right)$, and

$$
(f, g)=\sum_{n=0}^{m-1} f_{n} \bar{g}_{n}\left|a_{n}\right|, \quad[f, g]=\sum_{n=0}^{m-1} f_{n} \bar{g}_{n} a_{n}
$$

where $a_{0}, a_{1}, \ldots, a_{m-1}$ is a given real sequence of nonzero terms. The space $\mathbb{C}^{m}$ is then a Pontryagin space (basically, a finite-dimensional Krein space in this setting).

We define $\mathscr{I}$ be seeking out those vectors $f=\left(f_{n}\right) \in \mathbb{C}^{m}$ with real, positive components. Thus

$$
\mathscr{I}=\left\{f \in \mathbb{C}^{m}: f=\left(f_{0}, f_{1}, \ldots, f_{m-1}\right), f_{i}>0, i=0,1,2, \ldots, m-1\right\} .
$$

We define the "product" of two elements $f, g \in \mathbb{C}^{m}$ "componentwise"; thus, for $f=\left(f_{n}\right), g=\left(g_{n}\right)$ in $\mathbb{C}^{m}$, and $\alpha \in \mathbb{C}$,

$$
\alpha(f \circ g)=\left(\alpha f_{0} g_{0}, \alpha f_{1} g_{1}, \ldots, \alpha f_{m-1} g_{m-1}\right) \text {. }
$$

We say that $f \in \mathbb{C}^{m}$ is invertible if there is an element $f^{-1} \in \mathbb{C}^{m}$ such that

$$
f \circ f^{-1}=(1,1, \ldots, 1) \text {, }
$$

and it follows that $\mathscr{I}$ defined earlier is a class of invertible elements in the commutative algebra (with identity) just defined which is, in fact, also a Pontryagin space which we denote by $K$ once again. We delete the symbol "o" for simplicity. i.e.,

The symbol $\Delta f=\left(\Delta f_{n}\right)$ for $f \in K$ denotes the forward difference operator,

$$
\Delta f_{n}=f_{n+1}-f_{n}
$$

and $\Delta^{2} f=\left(\Delta^{2} f_{n}\right)=\left(\Delta\left(\Delta f_{n}\right)\right)$, etc. Let $b=\left(b_{0}, b_{1}, \ldots, b_{m-1}\right) \in K$. We define an operator $A$ on $K$ by

$$
(A f)_{n}=l\left(f_{n}\right)=\left\{-\Delta\left(\Delta f_{n-1}\right)+b_{n} f_{n}\right\} a_{n}
$$

reckon that $f_{-1}=0=f_{m}$ for $f \in K$ in the following calculations. Also note that $A$ is symmetric in $K$.

Theorem 3.2. For $f \in \mathscr{I}, A \in \mathscr{S}(f)$.

Proof. Let $u \in K, u \neq 0$. Then, using partial summation, we find

$$
[A u, u]=\sum_{n=0}^{m}\left\{\left|\Delta u_{n}\right|^{2}+b_{n}\left|u_{n}\right|^{2}\right\}+\left|u_{0}\right|^{2} \text {. }
$$

Furthermore,

$$
\left[(A f) f^{-1} I u, u\right]=\sum_{n=0}^{m}\left\{\frac{-\Delta\left(\Delta f_{n-1}\right)}{f_{n}}\left|u_{n}\right|^{2}+b_{n}\left|u_{n}\right|^{2}\right\}
$$


Thus

$$
\left[\left(A-(A f) f^{-1} I\right) u, u\right]=\left|u_{0}\right|^{2}+\sum_{n=0}^{m}\left|\Delta u_{n}\right|^{2}+\sum_{n=0}^{m} \frac{\Delta\left(\Delta f_{n-1}\right)}{f_{n}}\left|u_{n}\right|^{2} .
$$

Use of partial summation once again now gives

$$
\sum_{n=0}^{m} \frac{\Delta\left(\Delta f_{n-1}\right)}{f_{n}}\left|u_{n}\right|^{2}=\sum_{n=0}^{m} \Delta\left(\Delta f_{n-1}\right)\left(\frac{u_{n} \bar{u}_{n}}{f_{n}}\right)=-\left|u_{0}\right|^{2}-\sum_{n=0}^{m} \Delta\left(\frac{u_{n} \bar{u}_{n}}{f_{n}}\right) \Delta f_{n},
$$

and therefore

$$
\left[\left(A-(A f) f^{-1} I\right) u, u\right]=\sum_{n=0}^{m}\left|\Delta u_{n}\right|^{2}-\sum_{n=0}^{m} \Delta\left(\frac{u_{n} \bar{u}_{n}}{f_{n}}\right) \Delta f_{n} .
$$

Now use of the "difference of a product" formula shows that

$$
\Delta\left(\frac{u_{n} \bar{u}_{n}}{f_{n}}\right)=-\frac{\left|u_{n}\right|^{2}}{f_{n} f_{n+1}} \Delta f_{n}+\frac{\left|\Delta u_{n}\right|^{2}}{f_{n+1}}+\frac{1}{f_{n+1}}\left[\bar{u}_{n} \Delta u_{n}+u_{n} \Delta \bar{u}_{n}\right] .
$$

Combining the last two displays we obtain

$$
\begin{aligned}
{[(A-} & \left.\left.(A f) f^{-1} I\right) u, u\right] \\
& =\sum_{n=0}^{m}\left\{1-\frac{\Delta f_{n}}{f_{n+1}}\right\}\left|\Delta u_{n}\right|^{2}+\sum_{n=0}^{m}\left\{\frac{\left|u_{n}\right|^{2}}{f_{n} f_{n+1}}\left(\Delta f_{n}\right)^{2}-\frac{\Delta f_{n}}{f_{n+1}}\left[\bar{u}_{n} \Delta u_{n}+u_{n} \Delta \bar{u}_{n}\right]\right\} \\
& =\sum_{n=0}^{m} \frac{f_{n}}{f_{n+1}}\left\{\left|\Delta u_{n}\right|^{2}+\frac{\left|u_{n}\right|^{2}}{f_{n}^{2}}\left(\Delta f_{n}\right)^{2}-\frac{\Delta f_{n}}{f_{n}}\left[\bar{u}_{n} \Delta u_{n}+u_{n} \Delta \bar{u}_{n}\right]\right\} \\
& =\sum_{n=0}^{m} \frac{f_{n}}{f_{n+1}}\left(\Delta u_{n}-u_{n} \frac{\Delta f_{n}}{f_{n}}\right)\left(\Delta \bar{u}_{n}-\bar{u}_{n} \frac{\Delta f_{n}}{f_{n}}\right) \\
& =\sum_{n=0}^{m} \frac{f_{n}}{f_{n+1}}\left|\Delta u_{n}-u_{n} \frac{\Delta f_{n}}{f_{n}}\right|^{2}=\sum_{n=0}^{m} f_{n} f_{n+1}\left|\Delta\left(\frac{u_{n}}{f_{n}}\right)\right|^{2}=0
\end{aligned}
$$

if and only if $\left|\Delta\left(u_{n} / f_{n}\right)\right|=0$ for each $n$, since $f \in \mathcal{F}$, i.e., $u \in\langle f\rangle$, as desired.

Remark. The operator $A$ in Theorem 3.2 is represented by the tridiagonal matrix

$$
A=\left[\begin{array}{cccccc}
\left(b_{0}+2\right) / a_{0} & -1 / a_{0} & 0 & 0 & \cdots & 0 \\
-1 / a_{1} & \left(b_{1}+2\right) / a_{1} & -1 / a_{1} & 0 & \cdots & 0 \\
0 & -1 / a_{2} & & & \cdots & 0 \\
\vdots & \vdots & \vdots & \ddots & \cdots & \\
0 & 0 & \cdots & -1 / a_{m-1} & \cdots & \left(b_{m-1}+2\right) / a_{m-1}
\end{array}\right]
$$

Note that $A$ is not symmetric in the usual sense of the inner product on $\mathbb{C}^{m}$, but it is symmetric with respect to [ , ], i.e., as a matrix in the Pontryagin space $\mathbb{C}^{m}$. The proof of the next result is clear from Theorem 2.1.

Corollary 3.2. If the matrix $A$ has a nonreal eigenvalue, there is no real eigenvalue with a corresponding "positive" eigenvector.

Remark. The only assumptions on $A$ are those imposed at the outset, namely, that no $a_{i}$ vanishes and that not all $a_{i}$ 's are of the same sign (or else we cannot define a bona fide Pontryagin space). 
Clearly such matrices $A$ cannot be positive in the sense of Perron because of the sign condition on the $a_{i}$ 's. The corollary shows that there exist classes of matrices which are "very close to being positive" and for which the conclusion of the Perron-Fröbenius theorem fails. A similar remark may be formulated regarding the Krein-Rutman theorem. Of interest here is the fact that the presence of nonreal eigenvalues should not be incompatible with the existence of a Perron eigenvector (as is the case for nonnegative irreducible matrices), a phenomenon that cannot occur for the matrices considered here.

Example. The $3 \times 3$ matrix $A$ is defined by the rows $\{0,1,0\},\{-\varepsilon, 0, \varepsilon\}$, $\{0,1,0\}$, where $\varepsilon>0$ is not irreducible. However, $\lambda=0$ is always an eigenvalue and $\operatorname{col}(-1,0,1)$, is always an eigenvector (for every $\varepsilon>0$ ) which is not Perron. The limiting case $\varepsilon=0$ gives a nonnegative eigenvector (even though $A$ is reducible). For $\varepsilon>0$ the other two eigenvalues are nonreal and given by $\pm i \sqrt{2 \varepsilon}$. We have thus found a class of matrices $A(\varepsilon)$ such that $A(\varepsilon)$ tends to a nonnegative matrix with a Perron eigenvector, yet $A(\varepsilon)$ has no Perron eigenvector for any $\varepsilon>0$.

\section{ACKNOWLEDGMENTS}

The author acknowledges with thanks the support of the following institutions: the Natural Sciences and Engineering Research Council of Canada; the Dipartimenta di Matematica, Politecnico di Torino, Torino, Italy, for the kind hospitality rendered during the author's visit there. in May, 1992, at which time this article was conceived and written; and finally, the Consiglio Nazionale delle Ricerche, Italy.

\section{REFERENCES}

1. W. Allegretto and A. B. Mingarelli, Boundary problems of the second order with an indefinite weight-function, J. Reine Angew. Math. 398 (1989), 1-24.

2. $\ldots$ On the non-existence of positive solutions for a Schrödinger equation with an indefinite weight function, C. R. Math. Rep. Acad. Sci. Canada 8 (1986), 69-73.

3. F. V. Atkinson and A. B. Mingarelli, Asymptotics of the number of zeros and of the eigenvalues of general weighted Sturm-Liouville problems, J. Reine Angew. Math. 375/376 (1987), 380-393.

4. J. Bognár, Indefinite inner product spaces, Springer-Verlag, New York, 1974.

5. M. A. Krasnosel'skii, Positive solutions of operator equations, Noordhoff, The Netherlands, 1964.

Department of Mathematics and Statistics, Carleton University, Ottawa, Ontario, CANADA K1S 5B6

E-mail address: amingareøccs.carleton.ca 